\title{
A pictorial review of diseases causing meningeal enhance- ment in paediatric practice
}

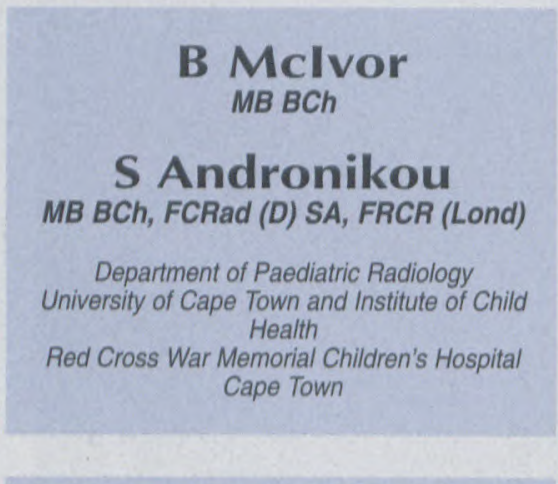

\section{Abstract}

Meningeal enhancement on contrastenhanced computed tomography (CT) and magnetic resonance imaging (MRI) scans can occur in normal or diseased meninges. Any process that causes meningeal irritation can cause abnormal meningeal enhancement. In certain clinical settings, abnormal meningeal enhancement can add weight to a suspected clinical diagnosis, e.g. tuberculous meningitis. However, in the setting of neoplastic disease, the presence of abnormal meningeal enhancement can imply metastatic involvement of the meninges, which alters patient management, and implies a worse prognosis. With previous surgery for brain parenchymal neoplasm, previous lumbar puncture, previous intrathecal chemotherapy, or craniospinal radio- therapy it is critical to distinguish between iatrogenic meningeal enhancement and metastatic involvement of the meninges. There are characteristics of the enhancement, which can be of assistance in making this differentiation. When abnormal meningeal enhancement is encountered unexpectedly, a differential diagnosis is provided to help elicit the cause.

\section{Introduction}

The purpose of this review is to demonstrate the array of pathology which results in enhancement of the meninges on contrast-enhanced computed tomography $(\mathrm{CT})$ and magnetic resonance imaging (MRI) scans of the brain and spine. It is also intended to aid in the interpretation of abnormal meningeal enhancement, as well as the absence of meningeal enhancement when meningeal disease is clinically suspected.

\section{Key concepts}

1. Any pathological process that causes meningeal irritation may result in meningeal enhancement on contrast-enhanced CT or MRI.

2. The absence of abnormal meningeal enhancement does not necessarily imply non-involvement of the meninges.

3. The presence of abnormal meningeal enhancement does not necessarily imply involvement of the meninges.

\section{Differential diag- nosis of abnor- mal meningeal enhancement}

Meningeal enhancement on contrast-enhanced MRI or less commonly CT scans may be seen in patients with normal meninges. The features of normal meningeal enhancement are linear, non-contiguous areas of enhancement, usually not seen on sequential slices. This results from uptake of contrast by the dura mater which lacks a blood-brain barrier.

In general terms, causes of abnormal meningeal enhancement can be classified using different criteria. The easiest classification is to group the causes into diseases that cause abnormal focal meningeal enhancement, and diseases that cause abnormal diffuse meningeal enhancement. Each of these groups can be further subdivided into conditions that occur commonly and uncommonly in clinical practice (Table I). ${ }^{2}$

However, the complete list of causes of abnormal meningeal enhancement is long, and an exhaustive list will not be given in this review.

\section{Anatomy of the meninges and extra-axial spaces}

An understanding of the anatomy of the meninges and extra-axial spaces is essential for the accurate interpretation of abnormal meningeal enhancement. ${ }^{1}$ 


\section{Meninges}

The meninges consist of three layers: dura mater, arachnoid mater and pia mater.

The dura has two layers. The outer layer forms the periosteum of the inner table of the skull, is very vascular, and is not of meningeal origin. The dura has no blood-brain barrier because the capillary endothelium has a discontinuous cell layer. Therefore, blood-borne metastases may access dura more easily than the arachnoid. The outer dural layer is not present in the spinal canal. ${ }^{1}$

The arachnoid is closely applied to the dura. It is thicker at the base of the brain than over the convexities. The arachnoid does have a bloodbrain barrier (capillary endothelial cells have tight junctions). ${ }^{1}$

The pia is a thin membrane which reaches into the depths of the cerebral sulci. Spinal pia is thicker than cerebral, and is more closely adherent to the underlying neural tissue.'

\section{Extra-axial spaces}

The epidural space forms when the dura becomes detached from the calvarium.

The subdural space is a potential space between the inner layer of the dura and the arachnoid. One author reports that the subdural space is not between the dura and arachnoid, but actually represents a cleft through the inner layer of the dura!

Both the epidural and subdural spaces are found only in pathological states.

The subarachnoid space lies between the arachnoid and pia, and contains cerebrospinal fluid (CSF) and vessels.

The same author reports that the Virchow-Robin spaces represent subpial spaces rather than a continuation of the subarachnoid space. ${ }^{1}$ This has consequences for the putative mechanism of spread of disease.

\section{Patterns of abnormal meningeal enhancement}

The pattern of abnormal meningeal enhancement can serve to direct the diagnosis towards a specific aetiology. Two patterns of abnormal
Table I. Aetiological classification of abnormal meningeal enhancement

\begin{tabular}{lc}
\hline Focal enhancement & Diffuse enhancement \\
\hline Common & Common \\
TB meningitis & Post surgery \\
Post surgery & Infectious meningitis \\
Metastases & Carcinomatous meningitis \\
Uncommon & Uncommon \\
Lymphoma/leukaemia & Subarachnoid haemorrhage \\
Meningioma & Dural sinus thrombosis \\
Dural cavernous haemangioma & Decreased intracranial pressure \\
Dural vascular malformation & Histiocytosis \\
Extramedullary haemopoiesis & Hypertrophic pachymeningitis \\
Histiocytosis & \\
Underlying infarct & \\
\hline
\end{tabular}

meningeal enhancement have been described:

1. Dura-arachnoid enhancement is curvilinear, runs immediately deep to the inner table of the skull, and along the falx and tentorium cerebelli. $^{3}$

2. Pia-subarachnoid enhancement closely follows the brain surface into the sulci and outlines the basal cisterns. Enhancement around the brain stem is always of the pia-arachnoid pattern.

The two patterns can occur simultaneously. The presence of a clearly defined pattern of enhancement impacts on the interpretation of subsequent investigations. It is important to remember that both inflammatory and neoplastic processes can cause both patterns of abnormal meningeal enhancement. ${ }^{1} \quad$ Diffuse involvement favours inflammatory causes, while nodular involvement is suggestive of a neoplastic cause.

\section{Imaging considerations}

The two major modalities used in the radiological evaluation of the meninges in children are contrastenhanced MRI and CT scans of the head and spine. In most instances MRI is the investigation of choice because of the absence of artefact from the overlying calvarium, and because of the multiplanar capabilities of MRI imaging. However, CT may play an important role in assessing bony changes of the calvarium or vertebral bodies. It must be borne in mind that a number of parameters of the MRI machine may alter the sensitivity of detection of meningeal enhancement. These include factors such as the magnet strength, the imaging sequence used, and the dose of 
contrast agent administered to the patient. The coronal plane is considered to be the optimal plane for visualisation of meningeal enhancement, although this depends on the site of the meningeal disease.

\section{Clinical images and discussion}

For the purposes of this review, the common causes of abnormal meningeal enhancement in clinical practice at the Red Cross War Memorial Children's Hospital (RXH) will be dealt with, followed by the less common causes. The three most common causes of abnormal meningeal enhancement at RXH are infection, neoplastic involvement of the meninges, and post-surgical enhancement.

The significance of the meningeal enhancement is dependent on the pathological cause. Abnormal meningeal enhancement resulting from infectious meningitis serves to strengthen the diagnosis of meningitis, but does not alter the management of the patient. The discovery of other complications of meningitis may affect patient management, e.g. hydrocephalus, subdural empyema or infarction.

However, in the presence of neoplasia, abnormal meningeal enhancement has significant implications for patient management and prognosis. In the setting of previous craniotomy or shunting, recent lumbar puncture, intrathecal chemotherapy or craniospinal irradiation, the distinction between neoplastic and iatrogenic enhancement is very important.

\section{Infection}

Meningeal infection of any aetiology may result in abnormal meningeal enhancement. The most common infectious cause of abnormal meningeal enhancement at RXH is tuberculous (TB) meningitis (Figs la - d).

TB meningitis causes gelatinous exudates in the basilar cisterns, resulting in a pia-subarachnoid enhancement pattern. The inflammatory process may result in a vasculitis that can give rise to ischaemia and infarction (Fig. 1c), usually in the lenticulostriate and thalamoperforating vessels. The imaging features of $\mathrm{TB}$ meningitis include basilar meningeal enhancement, as well as ependymal, cranial nerve and cerebral surface enhancement. Other commonly associated findings are hydrocephalus, parenchymal or meningeal tuberculomas (Fig. 1a), and occasionally increased density of the basal cisterns resulting from the gelatinous exudates. ${ }^{3}$ The usual enhancement pattern is pia-subarachnoid, which is

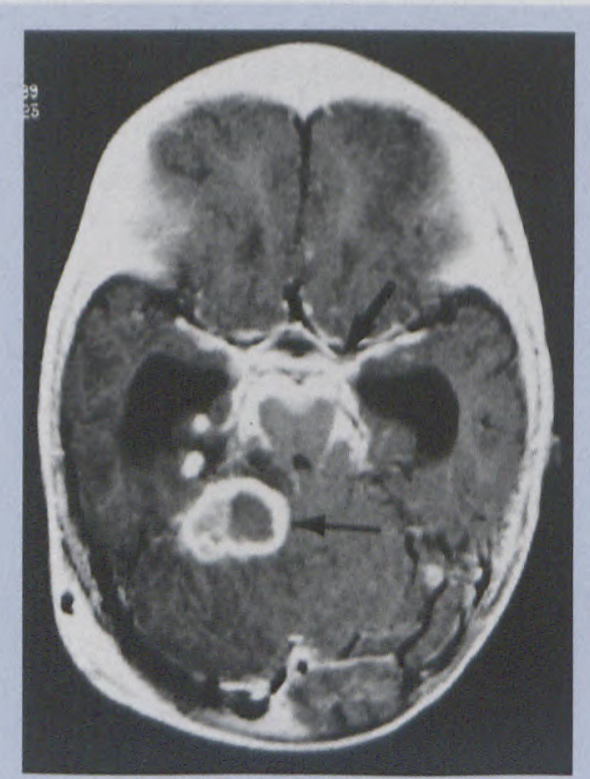

Fig. 1. Infectious meningeal disease.

Fig. 1a. Post-gadolinium T1-weighted axial MRI brain scan shows typical basal meningeal enhancement of the pia-subarachnoid pattern (thick arrow). A ring-enhancing cerebellar TB granuloma is also present (thin arrow). Hydrocephalus is seen $-a$ common imaging finding in TB meningitis. nodular, but diffuse enhancement is also possible.

Bacterial and viral meningitides

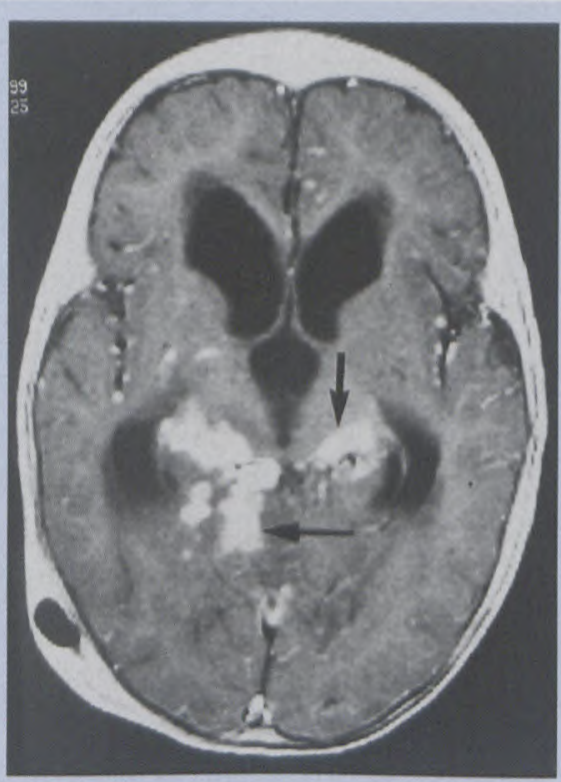

Fig. 1b. Post-gadolinium T1-weighted axial MRI brain scan showing nodular meningeal enhancement lining the quadrigeminal plate cistern asymmetrically, as well as the choroid fissures (thick arrow) and the tentorial hiatus (thin arrow). Hydrocephalus is also present.

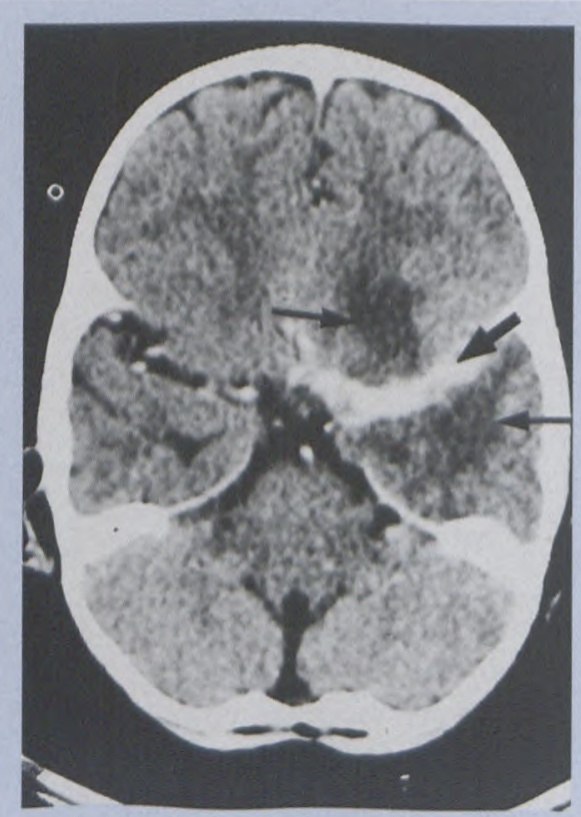

Fig. 1c. Contrast-enhanced axial CT scan of the brain demonstrating unilateral enhancement of the meninges lining the left middle cerebral artery cistern (thick arrow). Low densities in the frontal and temporal lobes represent areas of subacute infarction (thin arrows). 


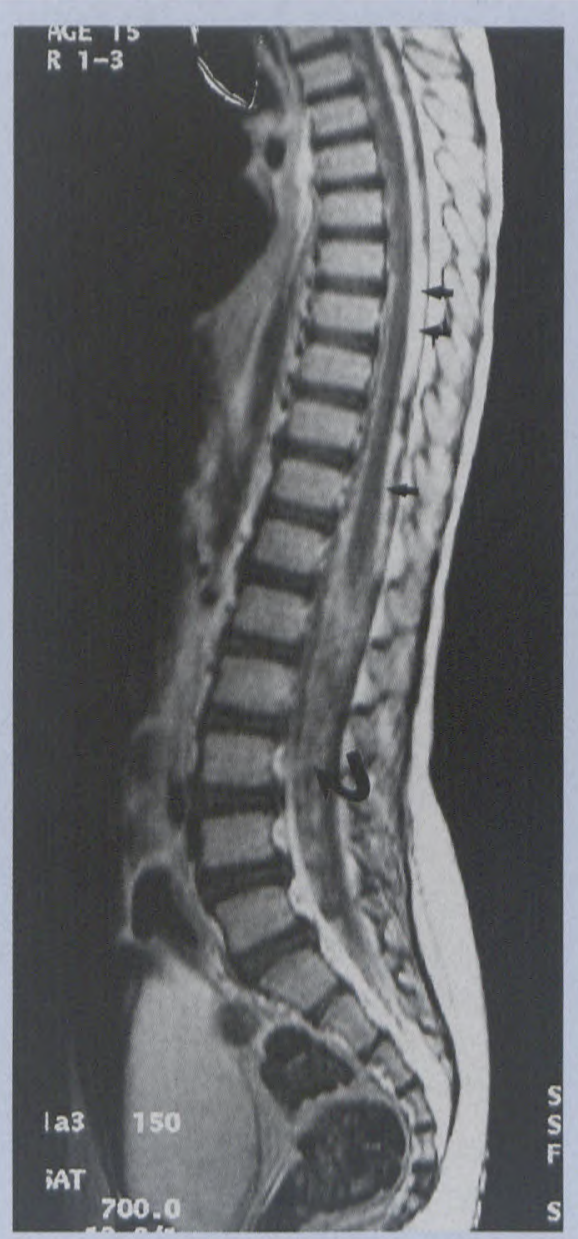

Fig. 1d. Post-gadolinium T1-weighted sagittal MR of the spine demonstrating thick irregular meningeal enhancement over the spinal cord (straight arrows) and shaggy enhancement involving the nerve roots of the cauda equina (curved arrow) in this patient with TB meningitis and spinal arachnoiditis.

less commonly cause abnormal meningeal enhancement, with less than $50 \%$ of cases of proven bacterial meningitis and even fewer cases of viral meningitis causing enhancement. ${ }^{3}$ There appears to be a critical degree of meningeal inflammatory reaction required before meningeal enhancement occurs, i.e. a threshold effect.' In AIDS patients, cytomegalovirus (CMV) and varicella zoster virus polyradiculomyelitis have been documented to cause diffuse enhancement of the cauda equina.

Other chronic meningitides also cause abnormal meningeal enhance-

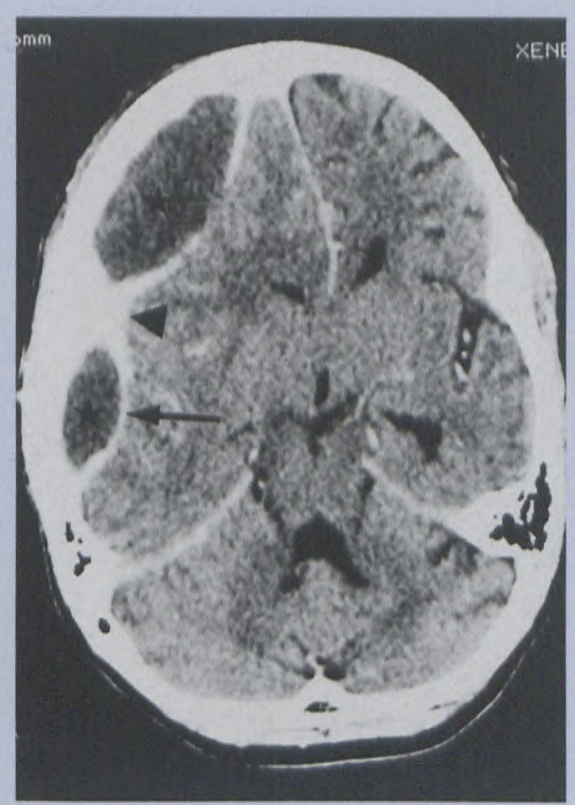

Fig. 1e. Contrast-enhanced axial CT scan of the brain showing a loculated subdural empyema (stars) with enhancement of the meninges medially (thin arrow), and adhesions (arrow head) in the right fronto-temporal region. The collection is causing mass effect and midline shift to the left.

ment. Cryptococcus neoformans meningitis in AIDS patients seldom results in abnormal meningeal enhancement because of a limited host inflammatory response. Aspergillus, seen primarily in recipients of organ transplants, can affect the central nervous (CNS). When this occurs, up to $20 \%$ of cases will demonstrate focal dura-arachnoid pattern thickening and enhancement. Neurosyphilis has been documented to cause localised and diffuse meningeal enhancement, ${ }^{1}$ but not in the paediatric age group.

Subdural empyema arising as a complication of paranasal sinusitis or other causes, also results in intense meningeal enhancement (Fig. 1e). ${ }^{3}$

\section{Neoplastic involvement of meninges}

Neoplastic involvement of the meninges in children is predominantly metastatic with primary meningeal tumours accounting for only $1-3 \%$ of primary CNS tumours. Metastatic meningeal involvement is becoming increasingly common because of the change in natural history (i.e. prolonged patient survival) of malignancies secondary to the improved treatment modalities.

Many tumours can spread to the meninges. Prior to the institution of CNS preventive therapy, the most common meningeal neoplastic process in children was due to acute lymphoblastic leukaemia. ${ }^{4}$ Metastases from primary CNS tumours are more common than metastases from nonCNS tumours, in contradistinction to the adult population. Examples of primary CNS neoplasms that metastasize to the meninges are medulloblastoma (Figs 2a, b, c), primitive neuroectodermal tumour (PNET), ependymoma, germ cell tumours, astrocytoma and glioblastoma. ${ }^{4}$

\section{Special investigations avail- able to diagnose neoplastic meningeal disease}

Metastatic neoplastic involvement of the meninges is diagnosed using a number of different investigations. CSF cytology is the gold standard for confirming the presence of meningeal metastases. It is positive (i.e. neoplastic cells detected in CSF), in up to $80 \%$ of patients after two samples, in metastatic meningeal involvement by nonCNS neoplasms. When the primary tumour is of CNS origin, the incidence of positive cytology in meningeal metastases is lower, at 12 $38 \%{ }^{1}$

Postmortem studies have shown that up to $41 \%$ of patients with metastases have negative antemortem CSF 


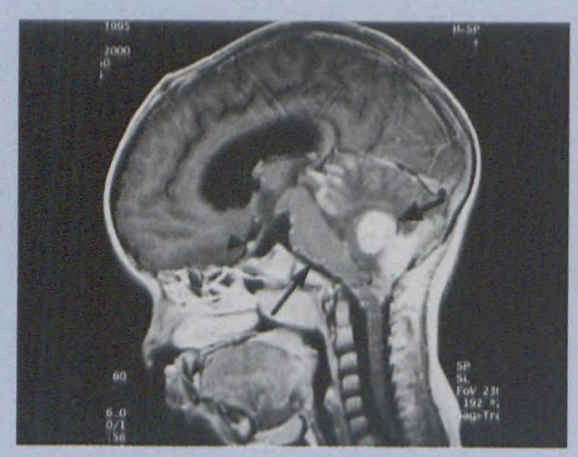

Fig. 2. Neoplastic meningeal disease. Fig. 2a. Post-gadolinium sagittal T1-weighted MRI of the brain demonstrating an enhancing posterior fossa mass (thick arrow) proven histologically to be a medulloblastoma. Note the continuous meningeal enhancement over the ventral aspect of the pons and medulla (thin arrow), as well as the enhancing nodule on the optic nerve (arrow head). This represents metastatic meningeal spread.

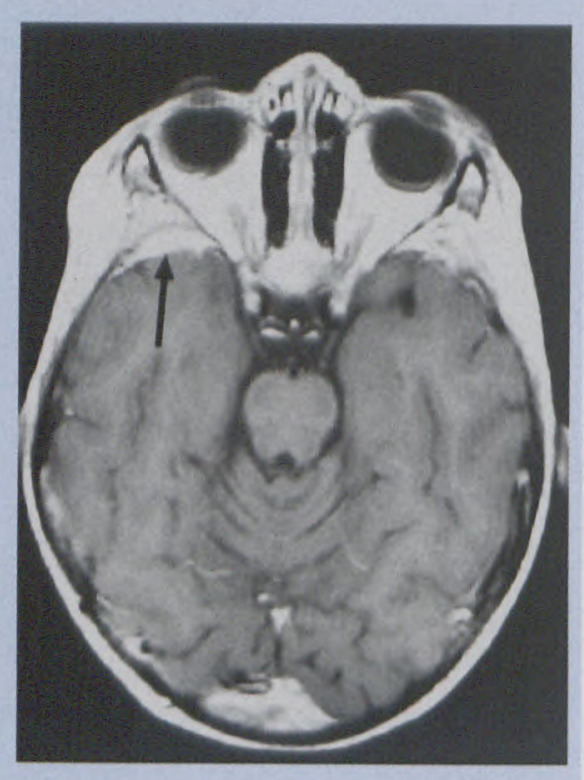

Fig. 2b. Post-gadolinium T1-weighted axial MRI of the brain demonstrating a plaque-like meningeal metastasis capping the anterior right temporal convexity (arrow), in a patient with proven medulloblastoma.

cytology. ${ }^{4}$ There are a number of reasons for this high false-negative rate. Focal meningeal metastases are less likely to yield positive CSF cytology than diffuse disease. ${ }^{1}$ When neoplastic involvement of the meninges is limited to the dura, this often results in negative CSF cytology. ${ }^{1}$ Dural disease will only yield positive cytology

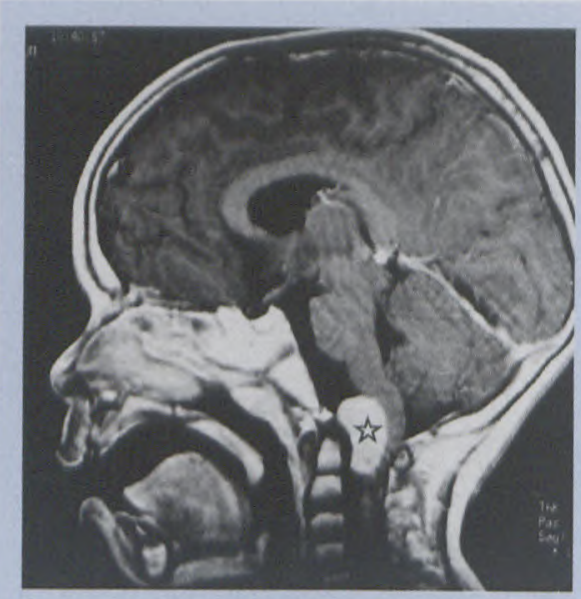

Fig. 2c. Post-gadolinium T1-weighted MRI of the brain demonstrating a brightly enhancing meningeal mass (star) anterior to the cervicomedullary junction, which was proven histologically to be a meningioma in this patient with neurofibromatosis type II.

once it has extended through the arachnoid and shed cells into the CSF. ${ }^{3}$ A higher rate of positive CSF cytology has been reported in the presence of the pia-subarachnoid enhancement pattern compared with the duraarachnoid pattern. ${ }^{1} \quad$ However, the presence of the dura-arachnoid enhancement pattern does not always imply that the subarachnoid space is spared. There is a small subgroup in whom CSF cytology remains persistently negative, and a positive result can only be obtained if a sample is taken from a CSF cistern or ventricle. ${ }^{4}$

When positive, CSF cytology makes the diagnosis of meningeal metastases. However, when CSF cytology is negative and meningeal metastases are suspected, other diagnostic modalities, namely MRI, radionuclide CSF flow studies and CSF chemistry come into play. Other CSF assays include tumour markers, and flow cytometry to identify malignant cells can also be used. Radionuclide CSF flow studies can be used to evaluate compartmentalisation of CSF. Although it does not demonstrate the meninges directly, it can demonstrate a CSF block that may render intrathecal chemotherapy ineffective. $^{4}$

\section{The role of imaging in meningeal metastatic disease}

When there are known meningeal metastases (from cytological examination of the CSF) contrast-enhanced MRI scans have the following role to play. Firstly, areas of bulky meningeal disease that are more susceptible to radiotherapy can be identified. CSF cytological examination cannot yield this information. In addition, the MRI scan is a non-invasive means of monitoring response to treatment of meningeal metastases. The MRI scan also identifies complications of meningeal metastases, e.g. hydrocephalus.

When meningeal metastases have not been proven (i.e. CSF cytology has not shown any malignant cells), the role of MRI changes. In this instance MRI in conjunction with other parameters, e.g. CSF chemistry and suggestive clinical signs (cranial nerve palsy, etc.), can be used to diagnose the presence of meningeal metastases. MRI can be used to direct meningeal biopsy for histological confirmation of metastasis, as well as to identify complications from meningeal metastasis and evaluate response to therapy.

\section{Limitations of MRI in the evaluation of meningeal disease}

It is important to note that MRI studies without intravenous (IV) contrast are insensitive to meningeal neoplasia. ${ }^{1}$ False-negatives are also common with contrast-enhanced MRI in one series there was a false-negative rate of $30 \%{ }^{1}$ This leads to the con- 
clusion that the absence of abnormal meningeal enhancement on MRI does not exclude meningeal involvement by metastatic neoplasia. In addition, MRI is less sensitive for meningeal involvement by haematological tumours (e.g. leukaemia) than for solid tumours.'

\section{MRI findings of meningeal metastases}

The main MRI findings of meningeal neoplasm are hydrocephalus, dura-arachnoid (Fig. 2b), pia-subarachnoid (Fig. 2a) and subependymal enhancement. Hydrocephalus is more likely in the presence of leptomeningeal invasion. While neoplastic meningeal involvement may be focal or diffuse, smooth or nodular, a nodular pattern is suggestive of (but not specific for) neoplastic involvement (Figs 3a-d). Nodular spinal metastases are often referred to as drop metastases (Fig. 3c). The imaging findings include diffuse pial enhancement that has been called 'sugar coating' (Fig. 3b). ${ }^{4}$ Pial metastases may appear to be intra-axial when situated in the depth of a Virchow-Robin space, or in the depth of a sulcus. The absence of parenchymal oedema and minimal mass effect are suggestive of an extra-axial lesion, i.e. on the cerebral surface. ${ }^{3}$ The clinical significance is that this is cerebral surface disease that may be amenable to intrathecal chemotherapy.

\section{Mechanisms of metastatic dural neoplastic involve- ment}

The dura can become involved by metastatic tumour via haematogenous spread, as it lacks a blood-brain barrier. Haematogenous spread from vertebral metastases via Batson's

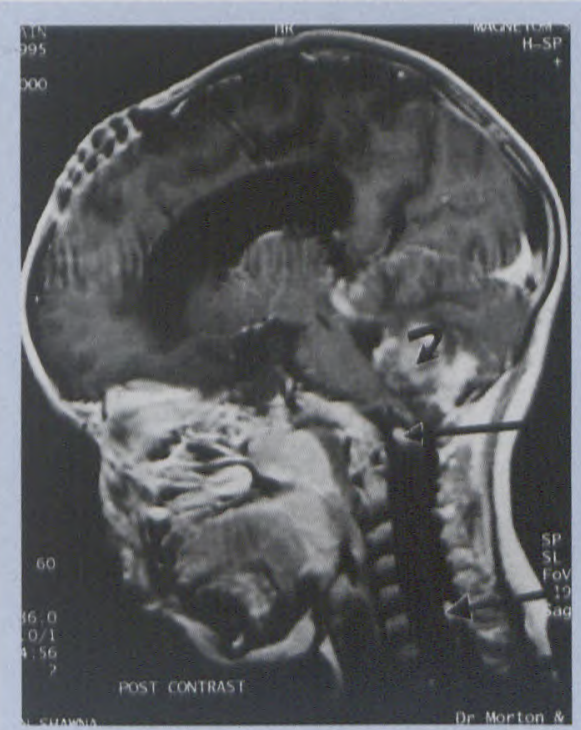

Fig. 3. Abnormal spinal meningeal enhancement.

Fig. 3a. Post-gadolinium T1-weighted sagittal MRI of the brain and spine demonstrating multifocal enhancing nodules over the spinal cord (straight arrows), representing drop metastases from a medulloblastoma (curved arrow).

plexus to the dural venous sinuses has been well documented. The other major mode of dural involvement by tumour is secondary to direct extension from the overlying bone. ${ }^{1,3}$

Leptomeningeal metastases can result from tumour spread to the choroid plexus which then sheds malignant cells into the CSF and the leptomeninges. ${ }^{1,3} \quad$ Choroid plexus metastases are often not appreciated on CT/MRI owing to the normal enhancement of the choroid plexus after intravenous contrast. A second mode of spread to the leptomeninges is from a parenchymal tumour through the vessels in the VirchowRobin spaces to the pia mater and then to the subarachnoid space. Other routes of spread include spread from dural neoplasms, direct extension from parenchymal tumours into leptomeninges, or from spinal epidural neoplasms along nerve routes or lymphatics. Once the CSF has been seeded, the neoplasm can spread

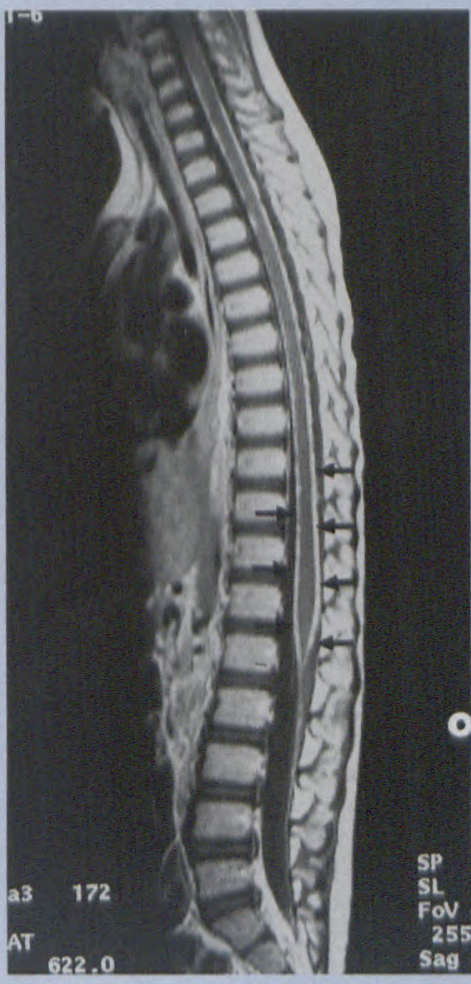

Fig. 3b. Post-gadolinium T1-weighted sagittal MRI of the spine demonstrating continuous, nodular meningeal enhancement (arrows) seen diffusely over the cord in this patient with meningeal spreac of medulloblastoma.

throughout the arachnoid, pia, Virchow-Robin spaces as well as the sheaths of the cranial and spinal nerves. Rarely, diffuse perivascular infiltration may result in ischaemic complications that are termed carcinomatous encephalitis.

\section{Importance of abnormal meningeal enhancement}

The recognition of abnormal meningeal enhancement is important in a number of different situations. In the presence of a malignant neoplasm, e.g. medulloblastoma, the detection of subtle meningeal enhancement or drop metastases implies a far worse prognosis for the patient (Fig. 3a). This may influence the surgical approach, and attempted resection of the primary tumour may be avoided in favour of biopsy and 


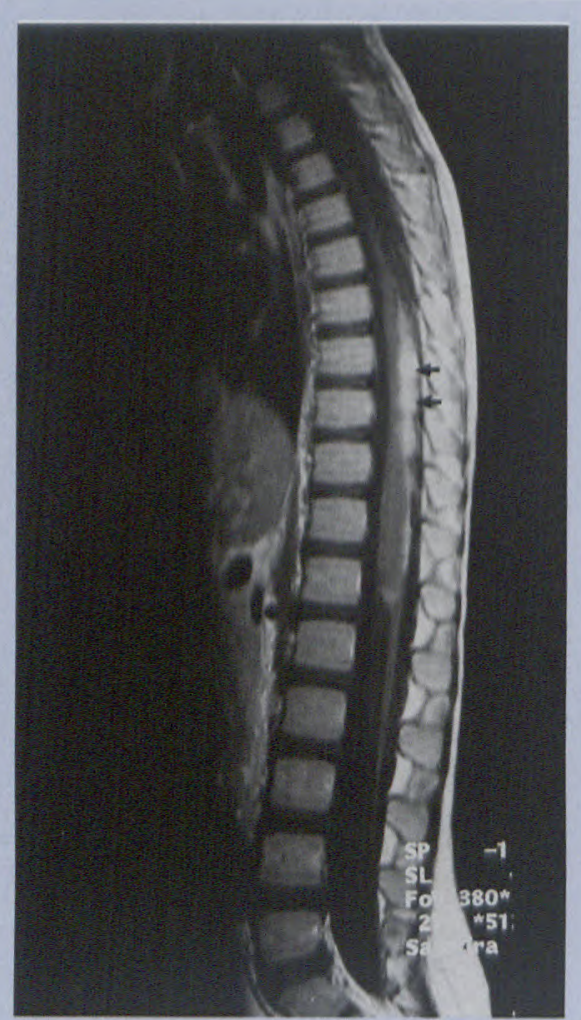

Fig. 3c. Post-gadolinium T1-weighted sagittal MRI of the spine demonstrating thick nodular meningeal enhancement over the thoracolumbar cord (arrows), and capping the conus medullaris representing meningeal spread of medulloblastoma.

palliative surgery, e.g. shunting, which has a lower morbidity. ${ }^{5}$ Another area of crucial importance is the differentiation between post-surgical meningeal enhancement and metastatic meningeal enhancement in the setting of a CNS tumour that has been surgically removed (see under 'Iatrogenic causes' for further discussion).

\section{Primary meningeal tumours}

Primary meningeal neoplasms are rare in children, accounting for $1-3 \%$ of primary paediatric CNS tumours. ${ }^{3}$ Approximately $25 \%$ of paediatric patients with meningioma suffer from neurofibromatosis type II (Fig. 2c). Cranial irradiation is also thought to be an aetiological factor in the development of meningioma. ${ }^{3}$ Meningiomas tend to be more aggressive in

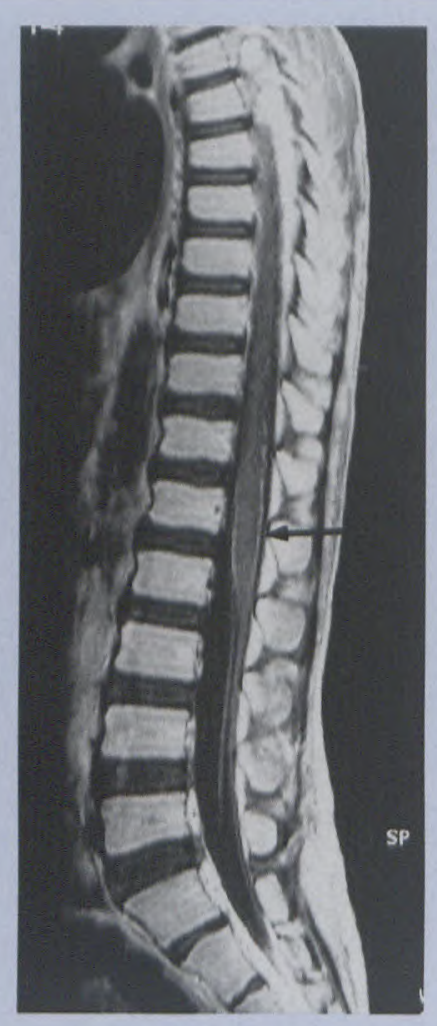

Fig. 3d. Post-gadolinium T1-weighted sagittal MRI of the spine demonstrating subtle meningeal enhancement over the conus medullaris (arrow), in a patient with acute lymphoblastic leukaemia.

children than in adults, and are more likely to occur in unusual places. ${ }^{3}$ Meningiomas are generally welldefined, lobulated mass lesions arising from the dura. On CT $60 \%$ are hyperdense, with up to $20 \%$ being calcified. On MR 60\% are hypointense on T1-weighted images. There is typically intense, homogeneous enhancement with the administration of intravenous contrast agent on CT and MRI. Certain studies have suggested a correlation between signal characteristics on T2-weighted images and histological subtypes, but this is controversial. ${ }^{3}$ A characteristic, but nonpathognomonic imaging feature of meningioma is the dural tail. This is an enhancing 'comet tail' extension of the dura adjacent to the tumour. It is important to note that the dural tail may indicate neoplastic involvement of the enhancing meninges, although the appearance is more commonly secondary to reactive changes in the adjacent meninges and not due to neoplastic involvement.

\section{latrogenic causes}

Surgery resulting in physical disruption of meningeal integrity (e.g. shunt/craniotomy) results in abnormal postoperative meningeal enhancement. Enhancement may be focal or diffuse (Fig. 4a). It is usually most marked in the presence of an extra-axial fluid collection (Fig. 4b), or if subarachnoid haemorrhage occurred at surgery. On CT scanning, abnormal post-surgical enhancement usually only lasts for several months. MRI, which is more sensitive for abnormal meningeal enhancement, may detect abnormalities for 1 - 2 years, but this enhancement usually regresses over time. ${ }^{1,3}$

In the setting of surgery for a primary CNS tumour, it may be important to differentiate between post-surgical meningeal enhancement and meningeal metastases that may have developed following surgery. Other laboratory tests, e.g. CSF cytology and chemistry may make the diagnosis of meningeal metastases. However, if these tests are equivocal, the imaging becomes important.

If the abnormal enhancement lies over the cerebral convexities and regresses over time, it is most likely post-surgical. However, if the enhancement is basal, of the 'nodular dural' or 'pia-subarachnoid' pattern, then this is suggestive of residual and/or recurrent neoplasm. Other abnormalities, e.g. recurrence of primary tumour, development of hydrocephalus, and other metastases would 


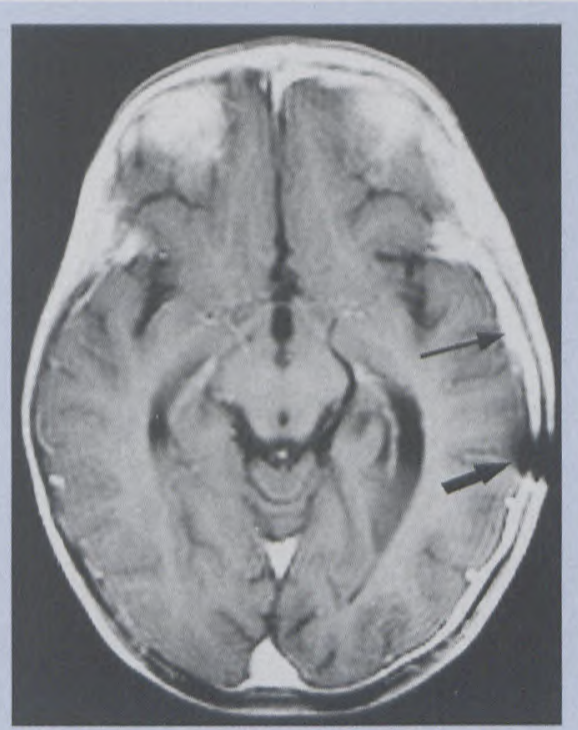

Fig. 4. latrogenic causes of abnormal meningeal enhancement.

Fig. 4a. Post-gadolinium T1-weighted axial MRI of the brain demonstrating an artefact caused by a metallic suture from a previous craniotomy in the left parietal region (thick arrow). Thick, continuous enhancement of the dura-arachnoid pattern noted in the underlying meninges (thin arrow) resulting from the previous surgery.

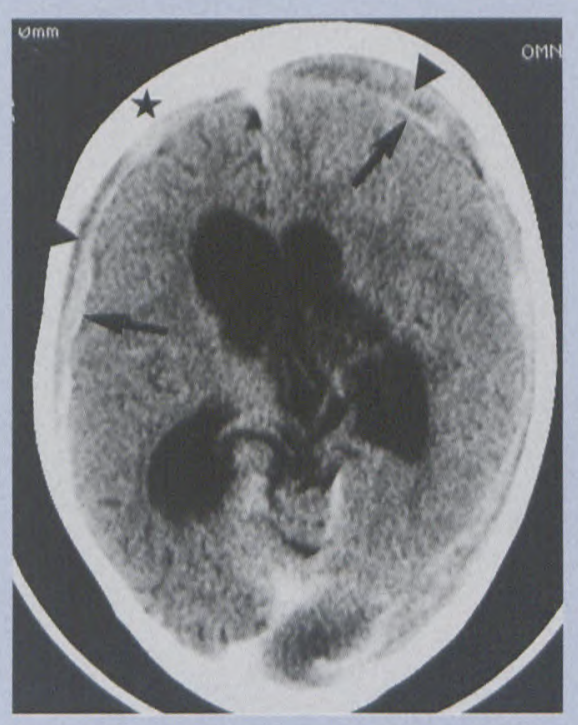

Fig. 4b. Contrast-enhanced axial CT scan of the brain demonstrating bilateral continuous meningeal enhancement (arrows), with bilateral isodense subdural collections (arrow heads), in this patient with complicated, chronic ventriculo-peritoneal shunt for hydrocephalus. Note the calvarial thickening (star) also associated with chronic shunting.

favour meningeal metastasis as the cause of the enhancement.

The other major iatrogenic cause of abnormal meningeal enhancement is lumbar puncture. The mechanism of enhancement is uncertain, but is thought to result from intra-cranial hypotension leading to meningeal hyperaemia, inflammation or nonspecific irritation, giving rise to smooth, diffuse meningeal enhancement. $^{3} \quad$ Other iatrogenic causes include intrathecal chemotherapy or craniospinal radiotherapy. ${ }^{2}$

\section{Post-traumatic/ vascular causes}

The presence of extra-axial collections often results in abnormal meningeal enhancement in the region of the collection. In addition, cranial trauma resulting in a CSF leak can cause diffuse meningeal enhancement, presumably secondary to intracranial hypotension. ${ }^{2}$ Subarachnoid haemorrhage can cause arachnoiditis resulting in generalised cisternal enhancement for up to several weeks after the initial bleed. ${ }^{2}$ MRI is notoriously inaccurate at detecting acute subarachnoid haemorrhage, sometimes resulting in mild $\mathrm{T} 1$ shortening of CSF giving rise to the dirty CSF' sign. Repeated subarachnoid haemorrhage can result in superficial siderosis, which has low signal intensity on T2-weighted images. ${ }^{1} \quad$ The meninges overlying an intraparenchymal haematoma can also occasionally demonstrate abnormal enhancement.

\section{Diseases that mimic meningeal disease}

Another consideration is the group of diseases that mimic abnormal meningeal enhancement. Such diseases affect the cerebral surface immediately deep to the meninges, or the calvarium.

Diseases causing gyriform enhancement simulating meningeal enhancement include Sturge-Weber syndrome, gliosarcoma, cerebral ischaemia, subpial tumour spread and a seizure focus.

In Sturge-Weber syndrome the large leptomeningeal angioma results in abnormal venous drainage from the underlying cerebral cortex. This causes ischaemia of the affected cortex, with focal atrophy and dystrophic calcification of the involved parenchyma (Fig. 5a). There is dilatation of the veins draining to the deep cerebral veins. ${ }^{2}$ The meningeal vascular malformation is seldom visualised on CT, being obscured by the overlying calvarium and cerebral calcification, but gyriform enhancement of the ischaemic cortex can be mistaken for abnormal meningeal enhancement. On MRI, however, the leptomeningeal angioma is not obscured by the calvarium and cortical calcification, and can be seen as an enhancing lesion (possibly resulting from slow blood flow), demonstrating the pia-arachnoid pattern of enhancement (Fig. 5b).

Subpial tumour spread includes the gliosarcoma, which is a rare primary CNS neoplasm that causes gyriform enhancement. This is difficult to distinguish from abnormal nodular meningeal enhancement (Fig. 5c).

Likewise, cerebral ischaemia or infarction may result in gyriform cortical enhancement that can be mistaken for meningeal enhancement. However, in large subacute supratentorial infarctions there can be duraarachnoid enhancement from days 2 to 6 following the infarct. The mechanism of this enhancement is unclear, but may result from reactive hyperaemia secondary to meningeal irritation, or to dilatation of collateral vessels. ${ }^{1,3}$ 
Gadopentetate dimeglumine
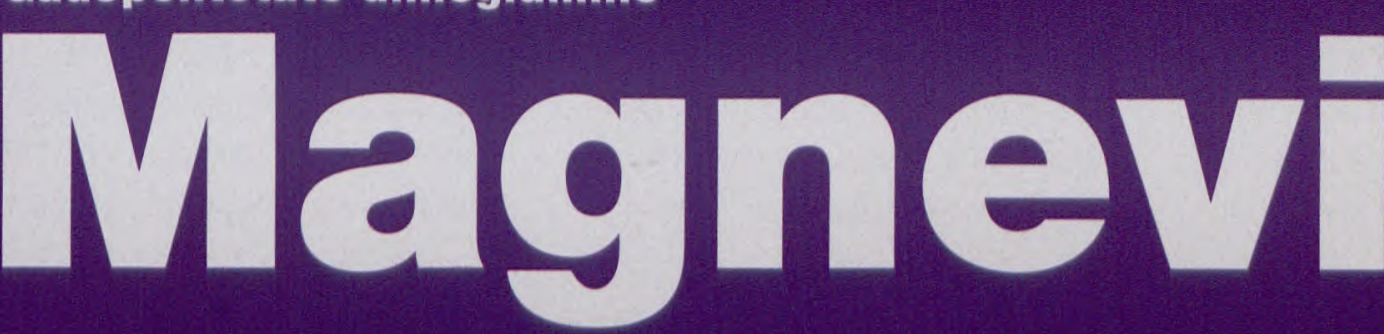


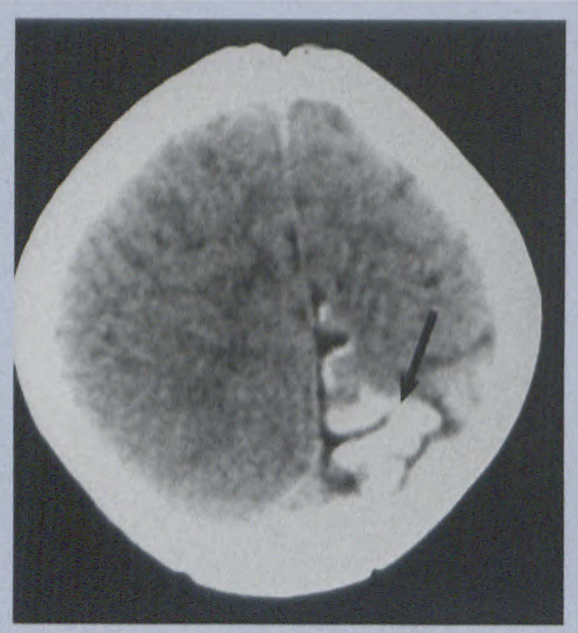

Fig. 5. Diseases that mimic meningeal disease. Fig. 5a. Non contrast-enhanced axial CT scan of the brain demonstrating gyriform hyperdensity in the left posterior parietal cerebral cortex (arrow) in this patient with Sturge-Weber syndrome. This is dystrophic calcification in ischaemic cerebral parenchy. ma.

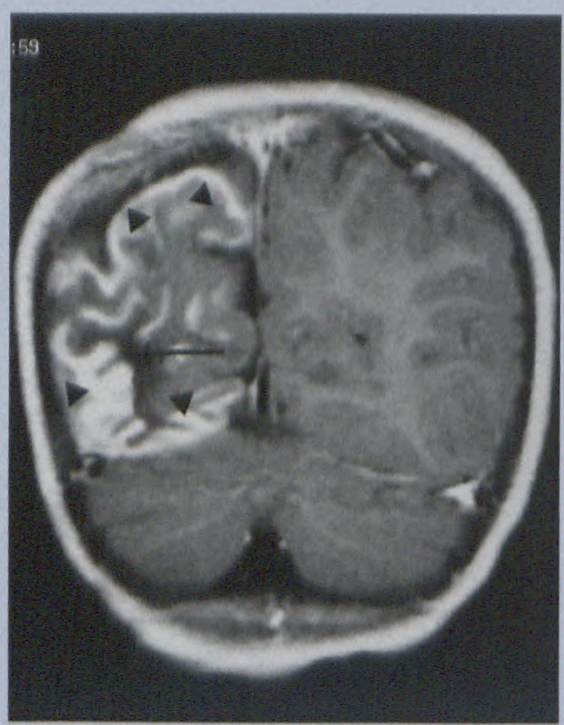

Fig. 5b. Post-gadolinium T1-weighted coronal MR scan of the brain in another patient with Sturge. Weber syndrome, demonstrating the leptomeningeal angioma as a continuous enhancing layer (arrow heads) of the pia-subarachnoid pattern overlying the low signal intensity calcified region (arrow) of the atrophic right occipital lobe.

The other compartment in which pathology mimics meningeal enhancement is the extradural space or bone overlying the meninges. Examples include calvarial tuberculosis (Figs $5 \mathrm{~d}$,e), and calvarial neuroblastoma metastases (Fig. 5f). Over time these diseases would invade the

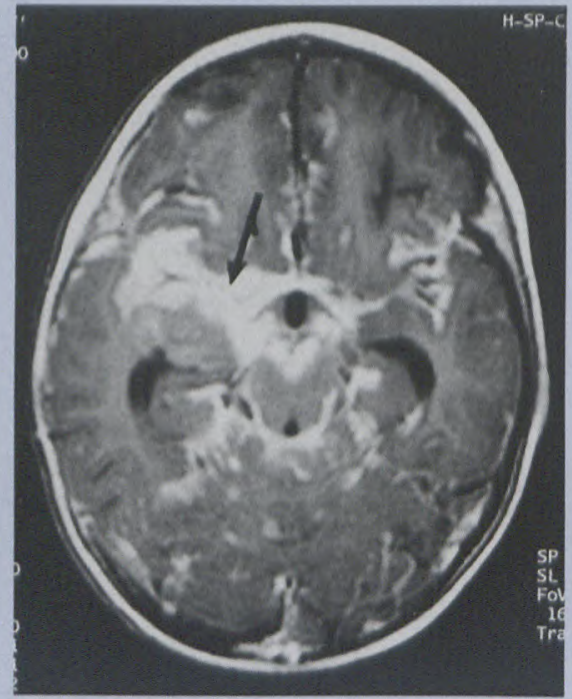

Fig. 5c. Post-gadolinium T1-weighted axial MRI scan of the brain demonstrating asymmetrical enhancement in what appears to be the basal meninges (arrow), but proven histologically to be parenchymal invasion by gliosarcoma.

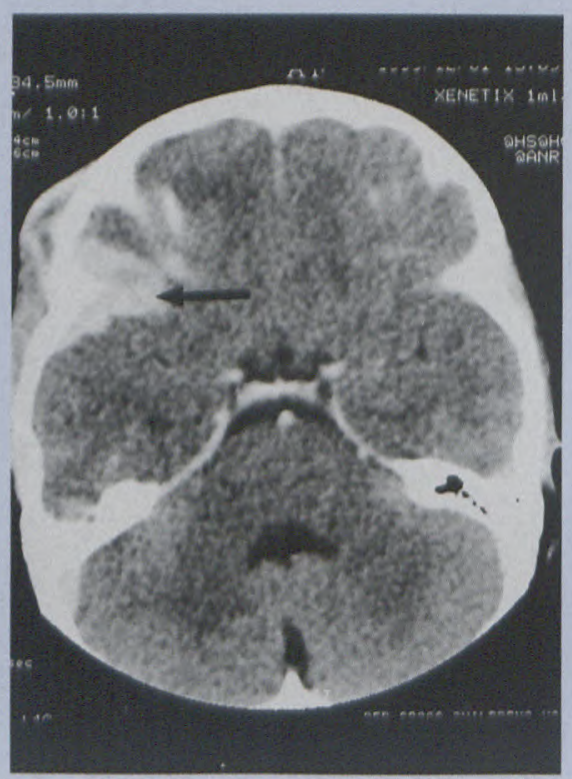

Fig. 5d. Contrast-enhanced axial CT scan of the brain demonstrating focal extra-axial enhancement deep to the right temporal bones (arrow) in this patient with calvarial TB.

meninges by contiguous spread resulting in abnormal meningeal enhancement.

\section{Conclusion}

This pictorial review is intended to illustrate the more common pathological processes resulting in abnor-

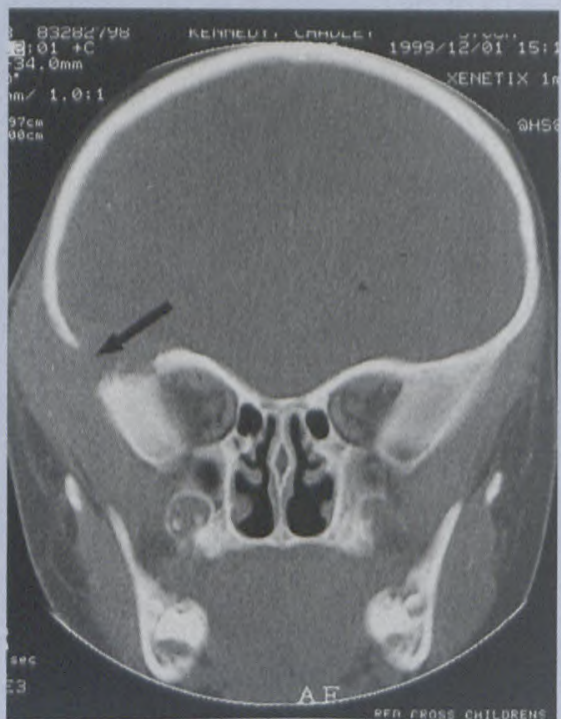

Fig. 5e. Coronal CT (bone algorithm) of patient in Fig. $5 d$ demonstrating the lytic bone lesion (arrow).

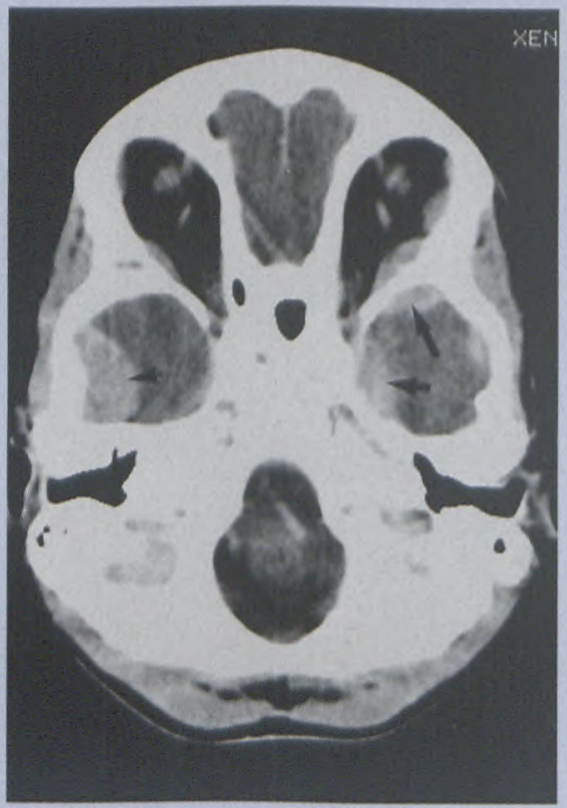

Fig. 5f. Contrast-enhanced axial CT scan of the brain demonstrating multifocal enhancing extraaxial nodules (arrows) representing calvarial metastases from a neuroblastoma, mimicking meningeal disease.

mal meningeal enhancement encountered in the paediatric population imaged at the RXH. In addition, a list of differential diagnoses is provided to aid in the interpretation of this finding. It is also intended to highlight the importance of detection of subtle abnormal meningeal enhancement in 
patients with malignant neoplasm, as the presence of meningeal metastases adversely affects the patient's prognosis and may alter subsequent management. It also emphasises the important role that imaging modalities have to play in distinguishing between abnormal enhancement caused by metastatic neoplasms and that arising from an iatrogenic aetiology.

\section{References}

1. Cidis Meltzer C, Fukui MB, Kanal E, et al. MR imaging of the meninges (Part I and Part II). Radiology 1996; 201: 297-306, 605-612.

2. Osborn AG. Brain tumors and tumorlike processes. In: Osborn AG, ed. Diagnostic Neuroradiology. St Louis: Mosby, 1994: 399-528.
3. Cinnamon J, Sharma M, Gray D, et al. Neuroimaging of meningeal disease. Semin Ultrasound CT MRI 1992; 15: 466-498.

4. Chamberlain MC. A review of leptomeningeal metastases in paediatrics. I Child Neurol 1995; 10: 191-199.

5. Akagami R, Steinbok P. Medulloblastoma with diffuse subarachnoid involvement. Pediatr Neurosurg 1999; 30: 53-54.

\section{Fundamentals of \\ Gastrointestinal Radiology \\ Michael Davis, and Jeffrey D. Houston}

This book provides a concise, yet thorough overview of the radiographic evaluation of a full range of commonly encountered gastrointestinal disease entities. For each segment of the gastrointestinal tract, readers will find a summary of the relevant radiographic anatomy, imaging techniques, and indications for radiographic examination... discussions of which imaging exam-inations are indicated for optimal evaluation of specific disease processes... and explanations of what to look for in the resultant images.

\section{Features}

$\checkmark$ Covers the functional and structural abnormalities of the entire alimentary tract, including the pharynx, esophagus, stomach, duodenum, small bowel, and colon, as well as the accessory organs of the gastrointestinal system, including the liver, biliary system, spleen, and pancreas.

$\checkmark$ Discusses all relevant imaging modalities - including conventional radiography, fluoroscopy, computed tomography, ultrasonography, magnetic resonance imaging, and nuclear medicine.

$\checkmark$ Includes over 395 examples demonstrating both normal and abnormal radiologic findings for easy comparison to the images seen in practice.

$\checkmark$ Utilises a straightforward, easy-to-read writing style to make complex material uncommonly accessible.

$\checkmark$ Recommended reading lists at the end of each chapter

Sept 2001, paperback, 240 pp, 298 illus., WBS, R480

\section{ORDERS}

The South African Medical Association Private Bag X1, Pinelands 7430

Tel (021) 530-6527, fax (021) 531-4126 E-mail: fpalm@samedical.org

Prepayment is required by cheque or Visa/Mastercard but not actioned until books are despatched. Please allow 3-4 weeks for delivery.

\section{Fundamentals of Fluoroscopy}

Jeffrey D. Houston and Michael Davis

A volume in the best-selling Fundamentals of Radiology series this compact resource equips readers to interpret fluoroscopic studies quickly and correctly. It offers thorough, hands-on guidance on all practical aspects of fluoroscopic imaging and interpretation. Conveniently organised by body system, this handy guide details a full range of non-interventional fluoroscopic procedures - both conventional and digital, common and rare. Features

$\checkmark$ Details non-interventional fluoroscopy for a full range of applications, including gastrointestinal, biliary, genitourinary, musculoskeletal, pediatric, and neuroradiology.

$\checkmark$ Provides coverage of indications and contraindications... patient preparation... equipment and supplies... recommended scout films... sample dictation... radiation dosage... and radiographic anatomy for each type of examination.

$\checkmark$ Features at-a-glance tables which guide the reader through image type (including film size and orientation), step-bystep procedural instructions, and tabletop and patient posi tioning.

$\checkmark$ Hundreds of real radiographic images demonstrate the desired results and capture nuances of technique.

$\checkmark$ Offers a systematic approach and engaging writing style that make fundamental fluoroscopic skills easy to master.

Sept 2001, paperback, 240 pp, 288 illustrations, WBS, R640

\section{Fundamentals of Pediatric Radiology}

\section{Lane F. Donnelly}

A basic primer covering fundamental information on paediatric imaging techniques and interpretation, for both radiology and paediatrics.

Features

$\checkmark$ Easily written for quick comprehension.

$\checkmark$ Short chapters cover core information for speedy access to key concepts

$\checkmark$ Excellent illustrations make the book useful.

$\checkmark$ Excellent chapters on paediatric chest, and on paediatric neuro-imaging

April 2001, paperback, WBS, R650 